PROCEEDINGS OF THE

AMERICAN MATHEMATICAL SOCIETY

Volume 125, Number 2, February 1997, Pages 407-416

S 0002-9939(97)03408-4

\title{
PROJECTIVE STRUCTURES ON MODULI SPACES OF COMPACT COMPLEX HYPERSURFACES
}

\author{
SERGEY MERKULOV AND HENRIK PEDERSEN
}

(Communicated by Christopher Croke)

\begin{abstract}
It is shown that moduli spaces of complete families of compact complex hypersurfaces in complex manifolds often come equipped canonically with projective structures satisfying some natural integrability conditions.
\end{abstract}

\section{Projective connections}

Let $M$ be a complex manifold. Consider the following equivalence relation on the set of affine torsion-free connections on $M$ : two connections $\widehat{\Gamma}$ and $\Gamma$ are said to be projectively equivalent if they have the same geodesics, considered as unparameterized paths. In a local coordinate chart $\left\{t^{\alpha}\right\}, \alpha=1, \ldots, \operatorname{dim} M$ on $M$, where $\hat{\Gamma}$ and $\Gamma$ are represented by Christoffel symbols $\hat{\Gamma}_{\alpha \beta}^{\gamma}$ and $\Gamma_{\alpha \beta}^{\gamma}$ respectively, this equivalence relation reads $[\mathrm{H}]$

$$
\widehat{\Gamma} \sim \Gamma
$$

if

$$
\hat{\Gamma}_{\alpha \beta}^{\gamma}=\Gamma_{\alpha \beta}^{\gamma} b_{\beta} \delta_{\alpha}^{\gamma}+b_{\alpha} \delta_{\beta}^{\gamma}
$$

for some 1-form $b=b_{\alpha} d t^{\alpha}$. An equivalence class of torsion-free affine connections under this relation is called a projective structure or a projective connection.

Let $M$ be a complex manifold with a projective structure A complex submanifold $P \subset M$ is called totally geodesic if for each point $t \subset P$ and each direction tangent to $P$ at $t$ the corresponding geodesic of the projective connection is contained in $P$ at least locally.

\section{Moduli SPACES of COMPACT COMPLEX hyPERSURFACES}

Let $X$ be be a compact complex hypersurface in a complex manifold $Y$ with normal line bundle $N$ such that $H^{1}(X, N)=0$. According to Kodaira [K-1], such a hypersurface $X$ belongs to the complete analytic family $\left\{X_{t} \mid t \in M\right\}$ of compact complex hypersurfaces $X_{t}$ in $Y$ with the moduli space $M$ being a $\left(\operatorname{dim}_{\mathbb{C}} H^{0}(X, N)\right)$ dimensional complex manifold. Moreover, there is a canonical isomorphism $k_{t}$ : $T_{t} M \rightarrow H^{0}\left(X_{t}, N_{t}\right)$ which associates a global section of the normal bundle $N_{t}$ of $X_{t} \hookrightarrow Y$ to any tangent vector at the corresponding point $t \in M$.

Received by the editors April 12, 1994 and, in revised form, April 13, 1995.

1991 Mathematics Subject Classification. Primary 32G10, 32L25, 53A15, 53B05, 53B10.

(C)1997 American Mathematical Society 
Consider $F=\left\{(y, t) \in Y \times M \mid y \in X_{t}\right\}$ and denote by $\mu: F \rightarrow Y$ and $\nu: F \rightarrow M$ two natural projections,

$$
Y \stackrel{\mu}{\leftarrow} F \stackrel{\nu}{\rightarrow} M .
$$

The space $F$ is a submanifold in $Y \times M$. If $N_{F}$ is the normal bundle of $F \hookrightarrow Y \times M$, then, for any point $t \in M$, we have $\left.N_{F}\right|_{\nu^{-1}(t)} \simeq N_{X_{t} \mid Y}$, where $N_{X_{t} \mid Y}$ is the normal bundle of the submanifold $\mu \circ \nu^{-1}(t)=X_{t} \hookrightarrow Y$. By Kodaira's theorem, there is an isomorphism $k: T M \rightarrow \nu_{*}^{0}\left(N_{F}\right)$, where $\nu_{*}^{0}\left(N_{F}\right)$ denotes the direct image.

Let us denote the point in the moduli space $M$ corresponding to $X$ by $t_{0}$, i.e $X=\mu \circ \nu^{-1}\left(t_{0}\right)$. It is easy to show that, for each $y \in Y^{\prime} \equiv \bigcup_{t \in M} X_{t}$ the set $\nu \circ \mu^{-1}(y)$ is a complex analytic subspace of $M$. We denote by $P_{y}$ its manifold content, i.e $P_{y}=\nu \circ \mu^{-1}(y) \backslash\{$ singular points $\}$. If the natural evaluation map

$$
\begin{aligned}
H^{0}\left(X_{t}, N_{X_{t} \mid Y}\right) & \rightarrow N_{z} \\
\phi & \rightarrow \phi(z),
\end{aligned}
$$

where $N_{z}$ is the fibre of $N$ at a point $z \in X_{t}$ and $\phi(z)$ is the value of the global section $\phi \in H^{0}\left(X_{t}, N_{X_{t} \mid Y}\right)$ at $z$, is surjective at all points $z \in X_{t}$ and for all $t \in M$, then $P_{y}=\nu \circ \mu^{-1}(y)$.

\section{THE MAIN THEOREM}

The idea of studying differential geometry on the moduli space of compact complex submanifolds of a given ambient complex manifold goes back to Penrose $[\mathrm{Pe}]$ who discovered self-dual conformal structures automatically induced on 4-dimensional moduli spaces of rational curves with normal bundle $N=\mathbb{C}^{2} \otimes \mathcal{O}(1)$. In this section we show that moduli spaces of compact complex hypersurfaces often come equipped canonically with induced projective structures satisfying some natural integrability conditions. Other manifestations of general and strong links between complex analysis and differential geometry can be found in Merkulov's survey $[\mathrm{M}]$.

Theorem 1. Let $X \hookrightarrow Y$ be a compact complex submanifold of codimension 1 with normal bundle $N$ such that $H^{1}(X, N)=0$ and let $M$ be the associated complete moduli space of relative deformations of $X$ inside $Y$. If $H^{1}\left(X, \mathcal{O}_{X}\right)=0$, then a sufficiently small neighbourhood $M_{0} \subset M$ of the point $t_{0} \in M$ corresponding to $X$, comes equipped canonically with a projective structure such that, for every point $y \in Y^{\prime} \equiv \bigcup_{t \in M} X_{t}$, the associated submanifold $P_{y} \subseteq \nu \circ \mu^{-1}(y) \cap M_{0}$ is totally geodesic.

Proof. An open neighbourhood of the submanifold $X \hookrightarrow Y$ can always be covered by a finite number of coordinate charts $\left\{W_{i}\right\}$ with local coordinate functions $\left(w_{i}, z_{i}^{a}\right), a=1, \ldots, n=\operatorname{dim} X$, on each neighbourhood $W_{i}$ such that $X \cap W_{i}$ coincides with the subspace of $W_{i}$ determined by the equation $w_{i}=0$. On the intersection $W_{i} \cap W_{j}$ the coordinates $w_{i}, z_{i}^{a}$ are holomorphic functions of $w_{j}$ and $z_{j}^{b}$,

$$
w_{i}=f_{i j}\left(w_{j}, z_{j}^{b}\right), \quad z_{i}^{a}=g_{i j}^{a}\left(w_{j}, z_{j}^{b}\right),
$$

with $f_{i j}\left(0, z_{j}^{b}\right)=0$. Here $z_{j}=\left(z_{j}^{1}, \ldots, z_{j}^{n}\right)$.

Let $U \subset M$ be a coordinate neighbourhood of the point $t_{0}$ with coordinate functions $t^{\alpha}, \alpha=1, \ldots, m=\operatorname{dim} M$. Then the coordinate domains $U \times W_{i}$ with 
coordinate functions $\left(w_{i}, z_{i}^{a}, t^{\alpha}\right)$ cover an open neighbourhood of $X \times U$ in the manifold $Y \times U$. For a sufficiently small $U$, the submanifold $F_{U} \cong \nu^{-1}(U) \hookrightarrow Y \times U$ is described in each coordinate chart $W_{i} \times U$ by an equation of the form [K-1]

$$
w_{i}=\phi_{i}\left(z_{i}, t\right),
$$

where $\phi_{i}\left(z_{i}, t\right)$ is a holomorphic function of $z_{i}^{a}$ and $t^{\alpha}$ which satisfies the boundary conditions $\phi_{i}\left(z_{i}, t_{0}\right)=0$. For each fixed $t \in U$ this equation defines a submanifold $X_{t} \cap W_{i} \hookrightarrow W_{i}$.

By construction, $F_{U}$ is covered by a finite number of coordinate neighbourhoods $\left\{V_{i} \equiv W_{i} \times\left. U\right|_{F}\right\}$ with local coordinate functions $\left(z_{i}^{a}, t^{\alpha}\right)$ which are related to each other on the intersections $V_{i} \cap V_{j}$ as

$$
z_{i}^{a}=g_{i j}^{a}\left(\phi_{j}\left(z_{j}, t\right), z_{j}\right)
$$

Obviously we have $\phi_{i}\left(g_{i j}\left(\phi_{j}\left(z_{j}, t\right), z_{j}\right), t\right)=f_{i j}\left(\phi_{j}\left(z_{j}, t\right), z_{j}\right)$.

TheKodaira map $k:\left.T M\right|_{U} \rightarrow \nu_{*}^{0}\left(\left.N_{F}\right|_{F_{U}}\right)$ can be described in the following way: take any vector field $v$ on $U$ and apply the corresponding 1st-order differential operator $V^{\alpha} \partial_{\alpha}$, where $\partial_{\alpha}=\partial / \partial t^{\alpha}$, to each function $\phi_{i}\left(z_{i}, t\right)$. The result is a collection of holomorphic functions $\sigma_{i}\left(z_{i}, t\right)=V^{\alpha} \partial_{\alpha} \phi_{i}\left(z_{i}, t\right)$ defined respectively on $V_{i}$. On the intersection $V_{i} \cap V_{j}$ one has $\left.\sigma_{i}\left(z_{i}, t\right)\right|_{z_{i}=g_{i j}\left(\phi_{j}, z_{j}\right)}=F_{i j}\left(z_{j}, t\right) \sigma_{j}\left(z_{j}, t\right)$, where

$$
\left.F_{i j} \equiv \frac{\partial f_{i j}}{\partial w_{j}}\right|_{w_{j}=\phi_{j}\left(z_{j}, t\right)}-\left.\left.\frac{\partial \phi_{i}}{\partial z_{i}^{a}}\right|_{z_{i}=g_{i j}\left(\phi_{j}, z_{j}\right)} \frac{\partial g_{i j}^{a}}{\partial w_{j}}\right|_{w_{j}=\phi_{j}\left(z_{j}, t\right)}
$$

is the transition matrix of the normal bundle $\left.N_{F}\right|_{F_{U}}$ on the overlap $F_{U} \cap V_{i} \cap V_{j}$. Therefore the 0 -cochain $\left\{\sigma_{i}\left(z_{i}, t\right)\right\}$ is a Čech 0-cocycle representing a global section $k(v)$ of the normal bundle $N_{F}$ over $F_{U}$.

Let us investigatehow second partial derivatives of $\left\{\phi_{i}\left(z_{i}, t\right)\right\}$ and $\left\{\phi_{j}\left(z_{j}, t\right)\right\}$ are related on the intersection $V_{i} \cap V_{j}$. Since

$$
\left.\frac{\partial \phi_{i}\left(z_{i}, t\right)}{\partial t^{\alpha}}\right|_{z_{i}=g_{i j}\left(\phi_{j}, z_{j}\right)}=F_{i j} \frac{\partial \phi_{j}\left(z_{j}, t\right)}{\partial t^{\alpha}}
$$

we find

$$
\left.\frac{\partial^{2} \phi_{i}}{\partial t^{\alpha} \partial t^{\beta}}\right|_{z_{i}=g_{i j}\left(\phi_{j}, z_{j}\right)}=F_{i j} \frac{\partial^{2} \phi_{j}}{\partial t^{\alpha} \partial t^{\beta}}+E_{i j} \frac{\partial \phi_{j}}{\partial t^{\alpha}} \frac{\partial \phi_{j}}{\partial t^{\beta}}-G_{i j \alpha} \frac{\partial \phi_{j}}{\partial t^{\beta}}-G_{i j \beta} \frac{\partial \phi_{j}}{\partial t^{\alpha}},
$$

where

$$
\begin{aligned}
E_{i j}= & \left.\frac{\partial^{2} f_{i j}}{\partial w_{j} \partial w_{j}}\right|_{w_{j}=\phi\left(z_{j}, t\right)}-\left.\left.\frac{\partial \phi_{i}}{\partial z_{i}^{a}}\right|_{z_{i}=g_{i j}\left(\phi_{j}, z_{j}\right)} \frac{\partial^{2} g_{i j}^{a}}{\partial w_{j} \partial w_{j}}\right|_{w_{j}=\phi_{j}\left(z_{j}, t\right)} \\
& -\left.\left.\frac{\partial^{2} \phi_{i}}{\partial z_{i}^{a} \partial z_{i}^{b}}\right|_{z_{i}=g_{i j}\left(\phi_{j}, z_{j}\right)}\left(\frac{\partial g_{i j}^{a}}{\partial w_{j}} \frac{\partial g_{i j}^{b}}{\partial w_{j}}\right)\right|_{w_{j}=\phi_{j}\left(z_{j}, t\right)},
\end{aligned}
$$

and

$$
G_{i j \alpha}=\left.\left.\frac{\partial^{2} \phi_{i}}{\partial z_{i}^{a} \partial t^{\alpha}}\right|_{z_{i}=g_{i j}\left(\phi_{j}, z_{j}\right)} \frac{\partial g_{i j}^{a}}{\partial w_{j}}\right|_{w_{j}=\phi_{j}\left(z_{j}, t\right)} .
$$

The collections $\left\{E_{i j}\right\}$ and $\left\{G_{i j \alpha}\right\}$ form 1-cochains with coefficients in $N_{F}^{*}$ and $\nu^{*}\left(\Omega^{1} M\right)$, respectively. Straightforward calculations reveal the obstructions for 
these two 1-cochains to be 1-cocycles,

$$
\begin{aligned}
\delta\left\{E_{i k}\right\} & =\left.2 \frac{\partial F_{i j}\left(z_{j}, t\right)}{\partial z_{j}^{a}} \frac{\partial g_{j k}^{a}}{\partial w_{k}}\right|_{w_{k}=\phi_{k}\left(z_{k}, t\right)}, \\
\delta\left\{G_{i k \alpha}\right\} & =\left.\frac{\partial F_{i j}\left(z_{j}, t\right)}{\partial z_{j}^{a}} \frac{\partial g_{j k}^{a}}{\partial w_{k}}\right|_{w_{k}=\phi_{k}\left(z_{k}, t\right)} \frac{\partial \phi_{j}\left(z_{j}, t\right)}{\partial t^{\alpha}} .
\end{aligned}
$$

From these equations we conclude that the 1-cochain $\left\{\tau_{i k \alpha}\right\}$, where

$$
\tau_{i k \alpha} \equiv \frac{1}{2} E_{i k} \frac{\partial \phi_{k}}{\partial t^{\alpha}}-G_{i k \alpha},
$$

is actually a 1-cocycle with values in $\nu^{*}\left(\Omega^{1} M\right)$. Since $H^{1}\left(X, \mathcal{O}_{X}\right)=0$, the semicontinuity principle [K-2] implies $H^{1}\left(X_{t}, \mathcal{O}_{X_{t}}\right)=0$ for all points in some Stein neighbourhood $M_{0} \subseteq U$. Hence, by the Leray spectral sequence

$$
H^{1}\left(\nu^{-1}\left(M_{0}\right), \nu^{*}\left(\Omega^{1} M\right)\right)=0 .
$$

Therefore, the 1-cocycle $\left\{\tau_{i k \alpha}\right\}$ is always a coboundary $\left\{\tau_{i j \alpha}\right\}=\delta\left\{\theta_{i \alpha}\right\}$, or more explicitly,

$$
\tau_{i j \alpha}\left(z_{j}, t\right)=F_{i j}\left(z_{j}, t\right)\left(-\left.\theta_{i \alpha}\left(z_{i}, t\right)\right|_{z_{i}=g_{i j}\left(\phi_{j}, z_{j}\right)}+\theta_{j \alpha}\left(z_{j}, t\right)\right),
$$

for some 0-cochain $\left\{\theta_{i \alpha}\left(z_{i}, t\right)\right\}$ on $\nu^{-1}\left(M_{0}\right)$ with values in $\nu^{*}\left(\Omega^{1} M\right)$. However, this 0 -cochain is defined non-uniquely-for any global section $\xi=\xi_{\alpha} d t^{\alpha}$ of $\nu^{*}\left(\Omega^{1} M\right)$ over $\nu^{-1}\left(M_{0}\right)$ the 0 -cochain

$$
\tilde{\theta}_{i \alpha}\left(z_{i}, t\right)=\theta_{i \alpha}\left(z_{i}, t\right)+\left.\xi_{\alpha}(t)\right|_{\nu^{-1}\left(M_{0}\right) \cap V_{i}}
$$

splits the same 1-cocycle $\left\{\tau_{i j \alpha}\right\}$. Note that, due to the compactness of the complex submanifolds $\nu^{-1}(t) \subset F$ for all $t \in M_{0}$ the components $\xi_{\alpha}$ of the global section $\xi \in H^{0}\left(\nu^{-1}\left(M_{0}\right), \nu^{*}\left(\Omega^{1} M\right)\right)$ are constant along the fibers, i.e. $\xi_{\alpha} \in \nu^{-1}\left(\mathcal{O}_{M_{0}}\right)$.

If we rewrite equation (2) in the form

$$
\begin{aligned}
\left.\frac{\partial^{2} \phi_{i}\left(z_{i}, t\right)}{\partial t^{\alpha} \partial^{\beta}}\right|_{z_{i}=g_{i j}\left(\phi_{j}, z_{j}\right)}= & F_{i j}\left(z_{j}, t\right) \frac{\partial^{2} \phi_{j}\left(z_{j}, t\right)}{\partial t^{\alpha} \partial t^{\beta}} \\
& +\tau_{i j \alpha}\left(z_{j}, t\right) \frac{\partial \phi_{j}\left(z_{j}, t\right)}{\partial t^{\beta}}+\tau_{i j \beta}\left(z_{j}, t\right) \frac{\partial \phi_{j}\left(z_{j}, t\right)}{\partial t^{\alpha}}
\end{aligned}
$$

and take equation (3) into account, we obtain the equality

$$
\left.\left(\frac{\partial^{2} \phi_{i}}{\partial t^{\alpha} \partial t^{\beta}}+\theta_{i \alpha} \frac{\partial \phi_{i}}{\partial t^{\beta}}+\theta_{i \beta} \frac{\partial \phi_{i}}{\partial t^{\alpha}}\right)\right|_{z_{i}=g_{i j}\left(\phi_{j}, z_{j}\right)}=\frac{\partial^{2} \phi_{j}}{\partial t^{\alpha} \partial t^{\beta}}+\theta_{j \alpha} \frac{\partial \phi_{j}}{\partial t^{\beta}}+\theta_{j \beta} \frac{\partial \phi_{j}}{\partial t^{\alpha}}
$$

which implies that, for each value of $\alpha$ and $\beta$, the holomorphic functions,

$$
\Phi_{i \alpha \beta}\left(z_{i}, t\right) \equiv \frac{\partial^{2} \phi_{i}\left(z_{i}, t\right)}{\partial t^{\alpha} \partial t^{\beta}}+\theta_{i \alpha}\left(z_{i}, t\right) \frac{\partial \phi_{i}\left(z_{i}, t\right)}{\partial t^{\beta}}+\theta_{i \beta}\left(z_{i}, t\right) \frac{\partial \phi_{i}\left(z_{i}, t\right)}{\partial t^{\alpha}},
$$

represent a global section of the normal bundle $N_{F}$ over $\nu^{-1}\left(M_{0}\right)$. Since the collections of functions $\left\{\partial_{\alpha} \phi_{i}\left(z_{i}, t\right)\right\}$ form a Čech representation of a basis for the free $\mathcal{O}_{M_{0}}$-module $\nu_{*}^{0}\left(\left.N_{F}\right|_{\nu^{-1}\left(M_{0}\right)}\right)$, the equality

$$
\Phi_{i \alpha \beta}\left(z_{i}, t\right)=\Gamma_{\alpha \beta}^{\gamma} \partial_{\alpha} \phi_{i}\left(z_{i}, t\right)
$$

must hold for some global holomorphic functions $\Gamma_{\alpha \beta}^{\gamma}$ on $\nu^{-1}\left(M_{0}\right)$. Since all the fibers $\nu^{-1}(t), t \in M_{0}$, are compact complex manifolds, these functions are actually pull-backs of some holomorphic functions on $M_{0}$. A coordinate system $\left\{t^{\alpha}\right\}$ on $M_{0}$ was used in the construction of $\Gamma_{\alpha \beta}^{\gamma}(t)$. However from (5) it immediately follows 
that under general coordinate transformations $t^{\alpha} \rightarrow t^{\alpha^{\prime}}=t^{\alpha^{\prime}}\left(t^{\beta}\right)$ these functions transform according to

$$
\Gamma_{\alpha^{\prime} \beta^{\prime}}^{\gamma^{\prime}}=\frac{\partial t^{\gamma^{\prime}}}{\partial t^{\delta}}\left(\Gamma_{\mu \nu}^{\delta} \frac{\partial t^{\mu}}{\partial t^{\alpha^{\prime}}} \frac{\partial t^{\nu}}{\partial t^{\beta^{\prime}}}+\frac{\partial^{2} t^{\delta}}{\partial t^{\alpha^{\prime}} \partial t^{\beta^{\prime}}}\right)
$$

Thus from any given splitting $\left\{\tau_{i j \alpha}\right\}=\delta\left\{\theta_{i \alpha}\right\}$ of the 1-cocycle $\left\{\tau_{i j \alpha}\right\}$ we extract a symmetric affine connection $\Gamma_{\alpha \beta}^{\gamma}(t)$. It is straightforward to check that this connection is independent of the choice of the $\left(w_{i}, z_{i}^{a}\right)$-coordinate system used in the construction and thus is well-defined except for the arbitrariness in its construction described by the transformations (4) which, as one can easily check, change the connection as follows:

$$
\begin{aligned}
\theta_{i \alpha}\left(z_{i}, t\right) & \rightarrow \theta_{i \alpha}\left(z_{i}, t\right)+\xi_{\alpha}(t) \\
\Gamma_{\alpha \beta}^{\gamma}(t) & \rightarrow \Gamma_{\alpha \beta}^{\gamma}(t)+\xi_{\alpha}(t) \delta_{\beta}^{\gamma}+\xi_{\beta}(t) \delta_{\alpha}^{\gamma} .
\end{aligned}
$$

Therefore we conclude that the neighbourhood $M_{0}$ of the point $t_{0}$ in the moduli space comes equipped canonically with a projective structure.

Let us now prove that for each point $y_{0} \in Y^{\prime}=\bigcup_{t \in M_{0}} X_{t}$, the associated submanifold $P_{y} \subseteq \nu \circ \mu^{-1}(y) \subset M_{0}$ is totally geodesic relative to the canonical projective connection in $M_{0}$. Suppose that $y_{0} \in W_{i}$ for some $i$. Then $y_{0}=\left(w_{i 0}, z_{i 0}^{a}\right)$ and the submanifold $P_{y_{0}}$ is given locally by the equations $w_{i 0}-\phi_{i}\left(z_{i 0}, t\right)=0$, where $t \in \nu \circ \mu^{-1}\left(y_{0}\right) \backslash$ singular points $\}$. Then a vector field $v(t)=\left.V^{\alpha} \partial_{\alpha}\right|_{P_{y_{0}}}$ is tangent to $P_{y_{0}}$ if and only if it satisfies the simultaneous equations

$$
V^{\alpha} \partial_{\alpha} \phi_{i}\left(z_{i 0}, t\right)=0 \text {. }
$$

In order to prove that the submanifold $P_{y_{0}}$ for arbitrary $y_{0} \in Y^{\prime}$ is totally geodesic relative to the canonical projective connection, we have to show that, for any vector fields $v(t)=V^{\alpha} \partial_{\alpha}$ and $w(t)=W^{\alpha} \partial_{\alpha}$ on $P_{y_{0}}$, the equation

$$
\left(W^{\beta} \partial_{\beta} V^{\alpha}+\Gamma_{\beta \gamma}^{\alpha} V^{\gamma} W^{\beta}\right) \bmod T P_{y_{0}}=0
$$

holds. Since $v(t)$ and $w(t)$ are tangent to $P_{y_{0}} \subset M$, we have the equation

$$
\begin{gathered}
W^{\beta}(t) \frac{\partial}{\partial t^{\beta}}\left(V^{\alpha} \partial_{\alpha} \phi_{i}\left(z_{i 0}, t\right)\right)=0, \\
V^{\alpha} W^{\beta} \frac{\partial^{2} \phi_{i}\left(z_{i 0}, t\right)}{\partial t^{\alpha} \partial t^{\beta}}=V^{\alpha} W^{\beta} \Gamma_{\alpha \beta}^{\gamma} \frac{\partial \phi_{i}\left(z_{i 0}, t\right)}{\partial t^{\gamma}} .
\end{gathered}
$$

From the latter equation and equation (8) it follows that

$$
\left(W^{\beta} \partial_{\beta} V^{\alpha}+\Gamma_{\beta \gamma}^{\alpha} V^{\gamma} W^{\beta}\right) \frac{\partial \phi_{i}\left(z_{i 0}, t\right)}{\partial t^{\alpha}}=0 .
$$

By (6) this means that $\left(W^{\beta} \partial_{\beta} V^{\alpha}+\Gamma_{\beta \gamma}^{\alpha} V^{\gamma} W^{\beta}\right) \partial_{\alpha} \in T P_{y_{0}}$, and thus equation (7) holds. The proof is completed.

We may have a moduli space even if the condition $H^{1}(X, N)=0$ is not satisfied. Given a moduli space, the proof above provides a projective structure so we have the following global result.

Corollary 2. Let $\left\{X_{t} \hookrightarrow Y \mid t \in M\right\}$ be a complete analytic family of compact complex hypersurfaces such that $H^{1}\left(X_{t}, \mathcal{O}_{X_{t}}\right)=0$ for all $t \in M$. Then the moduli space $M$ comes equipped canonically with a projective structure such that, for every point $y \in Y^{\prime}$, the associated submanifold $P_{y}=\nu \circ \mu^{-1}(y) \subset M$ is totally geodesic. 
We concludethis section with a brief geometric interpretation of geodesics canonically induced on moduli spaces of compact complex hypersurfaces. Any complex curve (immersed connected complex 1-manifold) in a complex manifold $M$ has a canonical lift to a complex curvein the projectivized tangent bundle $P_{M}(T M)$-one simply associates to each point of the curve its tangent direction. Then a projective structure on $M$ defines a family of lifted curves in $P_{M}(T M)$ which foliates the projectivized bundle holomorphically $[\mathrm{H}, \mathrm{L}]$. Then, for geodesically convex $M$, the quotient space of this foliation, $Z$, is a $(2 n-2)$-dimensional manifold, where $n=\operatorname{dim} M$. There is a double fibration

$$
Z \stackrel{\tau}{\leftarrow} P_{M}(T M) \stackrel{\sigma}{\rightarrow} M
$$

such that, for each $z \in Z, \sigma \circ \tau^{-1}(z) \subset M$ is a geodesic from the projectivestructure; for each $t \in M, \tau \circ \sigma^{-1}(t) \subset Z$ is projective space $\mathbb{C P}^{n-1}$ embedded into $Z$ with normal bundle $T \mathbb{C P}^{n-1}(-1)[\mathrm{L}]$.

Let $X_{0} \hookrightarrow Y$ be a compact complex submanifold of codimension 1 such that $H^{1}\left(X_{0}, N\right)=H^{1}\left(X_{0}, \mathcal{O}_{X_{0}}\right)=0$ and let $M$ be a geodesically convex domain in the associated complete moduli space of relative deformations of $X_{0}$ inside $Y$. The space of geodesics $Z$ can be identified in this case with the family of intersections $X_{s} \cap X_{t} \subset Y, s, t \in M$. From the explicit coordinate description of submanifolds $X_{t} \subset Y$ given in the proof of Theorem 1 one can easily see that, for each $t \in M$, the intersection $X_{t} \cap X_{0}$ is a divisor of the holomorphic line bundle on $X_{0}$ which is a holomorphic deformation of the normal bundle $N$. Since $H^{1}\left(X_{0}, \mathcal{O}_{X}\right)=0$, any holomorphic deformation of $N$ must be isomorphic to $N$ [K-3]. Therefore, each intersection $X_{t} \cap X_{0}$ is a divisor of the normal bundle on $X_{0}$, and, by completeness of the family $\left\{X_{t} \hookrightarrow Y \mid t \in M\right\}$, all divisors of $N$ arise in this way. If $t_{0} \in M$ is the point associated to $X_{0} \subset Y$ via the double fibration (1), then the set of all intersections $X_{0} \cap X_{t}$ is a projective space $\mathbb{C P}^{\operatorname{dim}}{ }^{M-1} \subset Z$ associated to $t_{0}$ via the double fibration (9). Then a geodesic through the point $t_{0} \in M$ is a family of $X_{t}$ which have the same intersection with $X_{0}$.

\section{Applichtions and examples}

One of the immediate applications of the theorem on projective connections is in the theory of 3-dimensional Einstein-Weyl manifolds. Hitchin $[\mathrm{H}]$ proved that there is a oneto-one correspondence between local solutions of Einstein-Weyl equations in 3 dimensions and pairs $(X, Y)$, where $Y$ is a complex 2-fold and $X$ is the projective line $\mathbb{C P}^{1}$ embedded into $Y$ with normal bundle $N \simeq \mathcal{O}(2)$. However the corresponding twistor techniques allowed one to compute only part of the canonical Einstein-Weyl structure induced on the complete moduli space $M$ of relative deformations of $X$ in $Y$, namely the conformal structure on $M$. Although the geodesics were formally described and the existence of a connection with special curvature was proved, no explicit formula for the connection was obtained. The theorem on projective connections fills this gap and provides one with a technique which is capable of decoding the full Einstein-Weyl structure from the holomorphic data of the embedding $X \hookrightarrow Y$. We shall use Theorem 1 in some examples to compute explicitly the canonical projective connection and then the canonical Einstein-Weyl structure on the complete moduli space of rational curves embedded into a 2-dimensional complex manifold with normal bundle $N \simeq \mathcal{O}(2)$. 
Consider a non-singular curve $X$ of bidegree $(1, n)$ in the quadric $\mathbb{C P}^{1} \times \mathbb{C P}^{1}$. Then $X$ is rational and has normal bundle $\mathcal{O}(2 n)[\mathrm{P}]$. The space $M$ of such curves can be described as follows: Let $(\zeta, \eta)$ be affine coordinates on $\mathbb{C P}^{1} \times \mathbb{C P}^{1}$ and consider the graph of a rational function of degree $n$ :

$$
\begin{gathered}
\eta=\frac{P(\zeta)}{Q(\zeta)}, \\
P(\zeta)=a_{n} \zeta^{n}+a_{n-1} \zeta^{n-1}+\cdots+a_{0}, \\
Q(\zeta)=b_{n} \zeta^{n}+b_{n-1} \zeta^{n-1}+\cdots+b_{0}
\end{gathered}
$$

The family of such $(1, n)$-curves is parameterized by $\mathbb{C P}^{2 n+1}$ and the space $M$ of non-singular curves is $\mathbb{C P}^{2 n+1} \backslash R$ where $R$ is the manifold of codimension 1 and degree $2 n$ given by the resultant of $P$ and $Q$. The geodesics of the projective connection are again given by projective lines in $\mathbb{C P}^{2 n+1} \backslash R$. We may of course choose to describe the induced structure on the hypersurface given by $R=1$, and for $n=1$ this corresponds to the standard projective structure on $S L(2, \mathbb{C})$ or on one of its real slices $\mathcal{H}^{3}, S^{3}$.

In order to obtain less trivial examples we consider branched coverings. Consider a complex curve $C$ contained in a complex surface $S$. We want to construct a branched covering of a neighbourhood of $C$ branched al ong $C$. Choose coordinates $\left(x_{i}, y_{i}\right)$ on neighbourhoods $O_{i}$ along $C$ such that $O_{i} \cap C$ is given by $x_{i}=0$. Then on overlaps we have $x_{i}=x_{j} H_{i j}\left(x_{j}, y_{j}\right)$ and $y_{i}=K_{i j}\left(x_{j}, y_{j}\right)$. Now, we look for an $n$-fold cover branched along $C$ : take patches $W_{i}$ with coordinates $\left(w_{i}, z_{i}\right)$ and define the covering map $\left(w_{i}, z_{i}\right) \rightarrow\left(x_{i}, y_{i}\right)=\left(w_{i}^{n}, z_{i}\right)$. This is a branched cover of $O_{i}$ branched along $O_{i} \cap C$. We want to identify the neighbourhoods $W_{i}$ along the curve $C$ to obtain a surface $Y$ with a map $\pi: Y \rightarrow S$ which locally has the form above. We get

$$
w_{i}^{n}=x_{i}=x_{j} H_{i j}\left(z_{j}, y_{j}\right)=w_{j}^{n} H_{i j}\left(w_{j}^{n}, z_{j}\right) .
$$

If we make a choice of the $n$-th root and put $\widetilde{H}_{i j}=H_{i j}^{1 / n}$ we get

$$
w_{i}=w_{j} \widetilde{H}_{i j}\left(w_{j}^{n}, z_{j}\right)=f_{i j}\left(w_{j}, z_{j}\right) \text {. }
$$

The obstruction for this to work along the curve is the class $\widetilde{H}_{i j} \widetilde{H}_{j k} \widetilde{H}_{k i} \in$ $H^{2}(C, \mathbb{Z} / n)$. We can identify this obstruction to be the self-intersection number of $C$ modulo $n$ : since $d x_{i}=H_{i j}\left(0, y_{j}\right) d x_{j}$ we see that $H_{i j}\left(0, y_{j}\right)$ represents the normal bundle $N$ in $H^{1}\left(C, \mathcal{O}^{*}\right)$. From the long exact sequence associated with

$$
0 \rightarrow \mathbb{Z} \rightarrow \mathcal{O} \stackrel{\exp }{\rightarrow} \mathcal{O}^{*} \rightarrow 0
$$

we see that the degree of $N$ is equal to $\log H_{i j}+\log H_{j k}+\log H_{k i}$. Thus, the obstruction to obtain $Y$ is equal to the self-intersection of $C$, modulo $n$. Each choice of $H_{i j}^{1 / n}$ corresponds to an element in $H^{1}(C, \mathbb{Z} / n)$. Unless the homology class of $C$ in $H_{2}(S, \mathbb{Z})$ is divisible by $n$ this local construction along the curve cannot be extended to work globally on $S[\mathrm{~A}]$.

Now, let us return to the case where $C$ is a $(1, n)$-curve in $\mathbb{C P}^{1} \times \mathbb{C P}^{1}$. In this case $C \cong \mathbb{C P}^{1}$, so there is a unique $n$-fold covering $Y$ branched along $C$ which we cannot extend to all of $\mathbb{C P}^{1} \times \mathbb{C P}^{1}$. The branch locus $X \subseteq Y$ is a copy of $C$ but $\operatorname{deg} N_{X}=\frac{1}{n} \operatorname{deg} N_{C}=2$ so we may describe an Einstein-Weyl structure on the moduli space of curves in $Y[\mathrm{H}]$ and contrary to earlier attempts we are now able to get the connection $\Gamma_{\alpha \beta}^{\gamma}$ explicitly. Let us concentrate on $(1,2)$ curves and let $C$ be 
the curve $\eta=\zeta^{2}$. The projection $\pi$ maps the curves in $Y$ onto those (1,2)-curves which meet $C$ in two points to second order. These curves may be given as in (10) with

$$
\begin{gathered}
P(\zeta)=\zeta^{2}-2 t_{0} t_{1} \zeta-t_{0}^{2}, \\
Q(\zeta)=t_{2}^{2} \zeta^{2}+2 t_{1} t_{2} \zeta+1+2 t_{0} t_{2}+t_{1}^{2}
\end{gathered}
$$

(see [P]). In order to describe the lifted curves we introduce the coordinates

$$
\begin{array}{ll}
x_{1}=\eta-\zeta^{2}, & x_{2}=\tilde{\eta}-\tilde{\zeta}^{2}, \\
y_{1}=\zeta, & y_{2}=\tilde{\zeta},
\end{array}
$$

where $(\tilde{\zeta}, \tilde{\eta})=\left(\frac{1}{\zeta}, \frac{1}{\eta}\right)$. Then $C$ is given by $x_{i}=0$. Making the coordinate transformation

$$
\begin{aligned}
& \left(x_{1}, y_{1}\right) \rightarrow(w, z)=\left(\sqrt{x_{1}}, y_{1}\right), \\
& \left(x_{2}, y_{2}\right) \rightarrow(\hat{w}, \hat{z})=\left(\sqrt{x_{1}}, y_{1}\right)
\end{aligned}
$$

we arrive at a covering of $Y$ by two coordinate charts $W$ and $\widehat{W}$ which is exactly of the type used in the proof of Theorem 1 and has the transition functions

$$
\hat{w}=f(w, z), \quad \hat{z}=g(z),
$$

given by

$$
f(w, z)=\frac{w}{z \sqrt{w^{2}+z^{2}}}, \quad g(z)=z^{-1}
$$

The complete maximal family of relative deformations of $C$ is described in this chart by the equations (in the notation of the proof of Theorem 1) $w=\phi(z, t)$ and $\hat{w}=\hat{\phi}(\hat{z}, t)$, with

$$
\phi(z, t)=i R(z) Q(z)^{-1 / 2}, \quad \hat{\phi}(z, t)=i R(z) P(z)^{-1 / 2},
$$

where $R(z)=t_{2} z^{2}+t_{1} z+t_{0}$. Note that a useful identity $P=z^{2} Q-R^{2}$ holds [P].

Now we have all the data to apply the machinery developed in the proof of Theorem 1. Following that scenario onefinds that thecanonical projectivestructure on $M$ can be represented by the following torsion-free affine connection

$$
\begin{array}{lll}
\Gamma_{01}^{0}=t_{1}\left(1+3 t_{0} t_{2}\right)(2 \Delta)^{-1}, & \Gamma_{01}^{1}=t_{2}\left(2+t_{1}^{2}+2 t_{0} t_{2}\right)(2 \Delta)^{-1}, \\
\Gamma_{00}^{0}=t_{2}\left(1+t_{0} t_{2}\right) \Delta^{-1}, & \Gamma_{00}^{1}=-t_{1} t_{2}^{2} \Delta^{-1}, \\
\Gamma_{02}^{0}=t_{0}\left(1+t_{0} t_{2}+t_{1}^{2}\right)(2 \Delta)^{-1}, & \Gamma_{02}^{1}=-t_{1}\left(1+t_{1}^{2}\right)(2 \Delta)^{-1}, \\
\Gamma_{11}^{0}=-t_{0}\left(1+t_{0} t_{2}\right) \Delta^{-1}, & \Gamma_{11}^{1}=t_{0} t_{1} t_{2} \Delta^{-1}, \\
\Gamma_{12}^{0}=-t_{0}^{2} t_{1}(2 \Delta)^{-1}, & \Gamma_{12}^{2}=-t_{0}\left(1+t_{0} t_{2}+t_{1}^{2}\right)(2 \Delta)^{-1}
\end{array}
$$

and all other Christoffel symbols being zero. Here

$$
\Delta=\left(1+t_{0} t_{2}\right)^{2}+t_{1}^{2}\left(1+2 t_{0} t_{2}\right) .
$$

Note that $\Delta^{2}=R$ where $R$ is the resultant of the polynomials in (10).

The conformal structure $[g]$ on $M$ is given by the condition for the curves to meet to second order. Thus we may choose the following metric in the conformal structure $[\mathrm{P}]$

$$
\begin{aligned}
g= & t_{1}^{2} t_{2}^{2} d t_{0}^{2}+\left(1+t_{0} t_{2}\right)^{2} d t_{1}^{2}+4 t_{0}^{2}\left(1+t_{1}^{2}\right) d t_{2}^{2}+2 t_{1} t_{2}\left(1+t_{0} t_{2}\right) d t_{0} d t_{1} \\
& -4\left(1+t_{1}^{2}\right)\left(1+t_{0} t_{2}\right) d t_{0} d t_{2}-4 t_{0}^{2} t_{1} t_{2} d t_{1} d t_{2} .
\end{aligned}
$$


Since our connection $\nabla$ is projectively equivalent to the Weyl connection $D$ it satisfies

$$
(\nabla g)_{\alpha \beta \gamma}=a_{\alpha} g_{\beta \gamma}+b_{\beta} g_{\alpha \gamma}+b_{\gamma} g_{\alpha \beta}
$$

for some 1-forms $a=\sum_{i=0}^{2} a_{\alpha} d t^{\alpha}$ and $b=\sum_{i=0}^{2} b_{\alpha} d t^{\alpha}$. We may solve these equations and present the Weyl connection $D$ in terms of the Levi-Civita connection $\nabla^{g}$ and the 1-form $\omega=a-2 b=\sum_{\alpha} \omega_{\alpha} d t_{\alpha}$,

$$
D=\nabla^{g}+\frac{1}{2} \omega^{\#} g-\omega \odot I
$$

see $[\mathrm{PT}]$. We get

$$
\begin{array}{ll}
a_{0}=3 t_{1}^{2} t_{2}(2 \Delta)^{-1}, & b_{0}=-3 t_{1}^{2} t_{2}(4 \Delta)^{-1} \\
a_{1}=-3 t_{1}\left(1+t_{0} t_{2}\right)(4 \Delta)^{-1}, & b_{1}=-3 t_{1}\left(1+t_{0} t_{1}\right)(4 \Delta)^{-1}, \\
a_{2}=-3 t_{0}\left(1+t_{0} t_{2}+t_{1}^{2}\right)(2 \Delta)^{-1}, & b_{2}=-3 t_{0}\left(1+t_{0} t_{2}+t_{1}^{2}\right)(2 \Delta)^{-1} .
\end{array}
$$

Thus using only the methods of the relative deformation theory of compact hypersurfaces we computed the full Einstein-Weyl structure on the moduli space.

Suppose we blow up a point $s$ on the quadric and take a $(1, n)$-curve passing through the point. Then in theblown up surface the curvewill haveself-intersection number $2 n-1$ and this corresponds to considering all the $(1, n)$-curves passing through $s$. We may combine this with the branched covering construction. In [PT] we considered the Einstein-Weyl structure associated to the $(1,3)$-curves: first we considered the 2-fold branched cover which reduced the degree of the normal bundle from 6 to 3 and then we blew up a point on the branch locus to get self-intersection equal to 2. Again we may compute the Weyl connection or compute the connection associated to any combination of blow up and branched cover. This will give non trivial examples with normal bundle $\mathcal{O}(n)$ for any $n$.

\section{ACKNOWLEDGMENTS}

It is a pleasure to thank Paul Tod for many valuable discussions and comments. Thanks are also due to Stephen Huggett, Yat Sun Poon and the anonymous referees for helpful remarks. One of the authors (SM) is grateful to the Department of Mathematics and Computer Science of Odense University for hospitality and financial support.

\section{REFERENCES}

[A] M. F. Atiyah, The signature of fibre bundles, in Global Analysis, Papers in honor of K. Kodaira (D. C. Spencer and S. Yanaga, eds.), Princeton Univ. Press, Princeton, 1969, 7384. MR 40:8071

[H] N. Hitchin, Complex manifolds and Einstein's equations, In: H. D. Doebner, et al. (eds.) Twistor geometry and non-linear systems, Lect. Notes Math., vol. 970, Springer-Verlag, Berlin, Heidelberg, New York, 1982, pp. 73-99. MR 84i:32041

[K-1] K. Kodaira, A theorem of completeness of characteristic systems for analytic families of compact submanifolds of complex manifolds, Ann. of Math. 75 (1962), 146-162. MR 24:A3665b

[K-2] Complex manifolds and deformations of complex structures, Springer-Verlag, New York, Berlin, Heidelberg, and Tokyo, 1986. MR 87d:32040

[K-3] K. Kodaira and D. C. Spencer, On deformations of complex analytic structures, I, Ann. of Math. 67 (1958), 328-401. MR 22:3009

[L] C. LeBrun, Spaces of complex geodesics and related structures, D. Phil. Thesis, Oxford University, 1980. 
[M] S. A. Merkulov, Relative deformation theory and differential geometry, In: S. A. Huggett, ed., Twistor Theory, Marcel Dekker, New York, 1995, pp. 107-132. MR 96b:32025

[P] H. Pedersen, Einstein-Weyl Spaces and $(1, n)$-Curves in the Quadric Surface, Ann. Global Anal. Geom. 4 (1986), 89-120. MR 88j:53045

[PT] H. Pedersen and K. P. Tod, Three-dimensional Einstein-Weyl Geometry, Adv. Math. 97 (1992), 74-109. MR 93m:53042

[Pe] R. Penrose, Non-linear gravitons and curved twistor theory, Gen. Rel. Grav. 7 (1976), 31-52. MR 55:11905

School of Mathematics and Statistics, University of Plymouth, Plymouth, Devon PL4 8AA, United Kingdom

Current address: Department of Pure Mathematics, University of Glasgow, 15 University Gardens, Glasgow G12 8QW, United Kingdom

Department of Mathematics and Computer Science, Odense University, Campusvej 55,5230 Odense M, Denmark 